\title{
STABLE FACTORIZATIONS OF SYMMETRIC TRIDIAGONAL AND TRIADIC MATRICES*
}

\author{
HAW-REN FANG ${ }^{\dagger}$ AND DIANNE P. O'LEARY ${ }^{\dagger}$
}

\begin{abstract}
We call a matrix triadic if it has no more than two nonzero off-diagonal elements in any column. A symmetric tridiagonal matrix is a special case. In this paper we consider $L X L^{T}$ factorizations of symmetric triadic matrices, where $L$ is unit lower triangular and $X$ is diagonal, block diagonal with $1 \times 1$ and $2 \times 2$ blocks, or the identity with $L$ lower triangular. We prove that with diagonal pivoting, the $L X L^{T}$ factorization of a symmetric triadic matrix is sparse, study some pivoting algorithms, discuss their growth factor and performance, analyze their stability, and develop perturbation bounds. These factorizations are useful in computing inertia, in solving linear systems of equations, and in determining modified Newton search directions.
\end{abstract}

Key words. matrix factorizations, tridiagonal matrices, pivoting, Cholesky decomposition

AMS subject classifications. 65F05, 65F50, 15A23

DOI. $10.1137 / 050636280$

1. Introduction. A symmetric matrix $A \in R^{n \times n}$ can be factored in the form $L X L^{T}$ in several ways:

1. $L L^{T}$ factorization with $L$ lower triangular and $X$ the identity.

2. $L D L^{T}$ factorization with $L$ unit lower triangular and $X$ diagonal.

3. $L B L^{T}$ factorization with $L$ unit lower triangular and $X$ block diagonal with block order 1 or 2 .

These $L X L^{T}$ factorizations can be used to solve linear systems $[1,3,4,6]$, to determine a downhill search direction in modified Newton methods [10,11], and to compute the inertia of a matrix [4].

Not all symmetric matrices have $L D L^{T}$ factorizations. We allow diagonal pivoting and factor $P A P^{T}$, where $P$ is a permutation matrix. With diagonal pivoting, we can ensure the existence of an $L B L^{T}$ factorization of any symmetric matrix and the existence of an $L D L^{T}$ factorization if $A$ is positive semidefinite or diagonally dominant. Diagonal pivoting is also used to improve numerical stability of the $L B L^{T}$ factorization when $A$ is indefinite $[1,3,4,6]$. Interchanging rows and columns can ruin the sparsity of $L X L^{T}$ factorizations of band matrices, so for tridiagonal matrices, attempts have been made to develop stable algorithms that do not require interchanges $[3,5,14]$.

In this paper, we study the sparsity and stability of $L X L^{T}$ factorizations for a class of symmetric matrices called triadic. A matrix $A$ is triadic if the number of nonzero off-diagonal elements in each column is bounded by 2 . Tridiagonal matrices are a special case of these, but other matrices, such as block diagonal matrices with full $3 \times 3$ blocks, and matrices that are tridiagonal except for entries in each corner, are also triadic. These latter matrices arise in the solution of differential equations with periodic boundary conditions.

${ }^{*}$ Received by the editors July 18, 2005; accepted for publication (in revised form) by P. Benner February 8, 2006; published electronically August 16, 2006. This work was supported by the National Science Foundation under grant CCR 02-04084.

http://www.siam.org/journals/simax/28-2/63628.html

${ }^{\dagger}$ Department of Computer Science and Institute for Advanced Computer Studies, University of Maryland, A. V. Williams Building, College Park, MD 20742 (hrfang@cs.umd.edu, oleary@cs.umd. edu). 
In section 2 we show that $L X L^{T}$ factorizations of a symmetric triadic matrix using diagonal pivoting remain sparse. Section 3 reviews various diagonal pivoting strategies for symmetric matrices, and they are applied to triadic matrices in section 4 . In section 5 the perturbation analysis of these factorizations is discussed. Section 6 gives conclusions. A rounding error analysis for these factorizations is given in [8], which also includes analysis when $A$ is rank-deficient.

One application of $L X L^{T}$ factorizations of triadic matrices is in modified Cholesky algorithms to safeguard the Newton method. Modified Cholesky algorithms replace the Hessian matrix $A$ by $A+E$, for a suitably chosen perturbation matrix $E$, in order to ensure that we are factoring a positive definite matrix and therefore computing a downhill search direction. In a subsequent paper, we will discuss the usefulness of triadic matrices in such algorithms [9].

2. Diagonal pivoting in $L X L^{T}$ factorization preserves triadic structure. In this section, we show that diagonal pivoting preserves sparsity in the $L X L^{T}$ factorizations of symmetric triadic matrices. This is a consequence of the property that for any permutation matrix $P, P A P^{T}$ is symmetric triadic if and only if $A$ is symmetric triadic.

First we consider the sparsity of $L D L^{T}$ (and thus $L L^{T}$ ) factorizations. The following lemma on the structure of the Schur complements leads to the desired result. We define $e_{k}$ to be the column vector that is zero except for a 1 in its $k$ th position.

Lemma 2.1. Let $A=\left[\begin{array}{cc}a_{11} & c_{1}^{T} \\ c_{1} & A_{22}\end{array}\right]$ be a symmetric triadic matrix with $a_{11} \neq 0$. Then the Schur complement $\bar{A}=A_{22}-c_{1} c_{1}^{T} / a_{11}$ is symmetric triadic.

Proof. Since $A$ is triadic, $c_{1}$ has at most two nonzero elements. We denote them by $c_{i 1}=\xi$ and $c_{j 1}=\eta$. The matrix $A_{22}$ is also triadic and its $i$ th and $j$ th rows have at most one off-diagonal element each. Moreover,

$$
c_{1} c_{1}^{T}=\xi^{2} e_{i} e_{i}^{T}+\xi \eta\left(e_{i} e_{j}^{T}+e_{j} e_{i}^{T}\right)+\eta^{2} e_{j} e_{j}^{T}
$$

has at most four nonzero elements. Two of these are on the diagonal, and the others are in positions $(i, j)$ and $(j, i)$. Thus the sum of $A_{22}$ and $-c_{1} c_{1}^{T} / a_{11}$ is triadic.

THEOREM 2.2. In the $L D L^{T}$ factorization of a symmetric triadic matrix, $L$ is triadic.

Proof. The proof is by finite induction. At the $k$ th step, assume that the remaining $(n-k+1) \times(n-k+1)$ matrix $\bar{A}$ is symmetric triadic. Then the next column of $L$ is computed as $c_{1} / a_{11}$, where

$$
\bar{A}=\left[\begin{array}{cc}
a_{11} & c_{1}^{T} \\
c_{1} & A_{22}
\end{array}\right]=\left[\begin{array}{cc}
1 & 0 \\
c_{1} / a_{11} & I
\end{array}\right]\left[\begin{array}{cc}
a_{11} & 0 \\
0 & \tilde{A}
\end{array}\right]\left[\begin{array}{cc}
1 & c_{1}^{T} / a_{11} \\
0 & I
\end{array}\right]
$$

and $\tilde{A}=A_{22}-c_{1} c_{1}^{T} / a_{11}$ is the Schur complement of $\bar{A}$. Notice that $c_{1}$ has at most two elements. By Lemma 2.1, the matrix $\tilde{A}$, which becomes $\bar{A}$ for the next iteration, is triadic, so we can continue the induction.

Now we establish the same result for the $L B L^{T}$ factorization. The algorithm for $L B L^{T}$ factorization is the same as for $L D L^{T}$ factorization with diagonal pivoting, except when all diagonal elements of the Schur complement are zeros. In such a case, we diagonally pivot some nonzero off-diagonal element in the lower triangular part to be at the second row and first column in the Schur complement and pivot with respect to the $2 \times 2$ block. This decomposition can be used to control element growth for numerical stability, even if we find a nonzero diagonal element $[1,3,4,6]$. 
Lemma 2.3. Let $A=\left[\begin{array}{ll}A_{11} & A_{21}^{T} \\ A_{21} & A_{22}\end{array}\right]$ be a symmetric triadic matrix, where $A_{11}=$ $\left[\begin{array}{cc}\sigma_{1} & a \\ a & \sigma_{2}\end{array}\right], a \neq 0$, and $\operatorname{det}\left(A_{11}\right) \neq 0$. Then the Schur complement $\bar{A}=A_{22}-A_{21} A_{11}^{-1} A_{21}^{T}$ with respect to the $2 \times 2$ pivot $A_{11}$ is symmetric triadic.

Proof. Since $\operatorname{det}\left(A_{11}\right) \neq 0, A_{11}^{-1}=\frac{1}{\operatorname{det}\left(A_{11}\right)}\left[\begin{array}{cc}\sigma_{2} & -a \\ -a & \sigma_{1}\end{array}\right]$. Since $A$ has at most two nonzero off-diagonal elements in each column and $A_{11}$ already has one nonzero offdiagonal element in each column, $A_{21}$ has at most one nonzero element in each column, so we denote it as $A_{21}=\left[\begin{array}{ll}\xi e_{i} & \eta e_{j}\end{array}\right]$. Then

$$
\begin{aligned}
A_{21} A_{11}^{-1} A_{21}^{T} & =\frac{1}{\operatorname{det}\left(A_{11}\right)}\left[\begin{array}{ll}
\xi e_{i} & \eta e_{j}
\end{array}\right]\left[\begin{array}{cc}
\sigma_{2} & -a \\
-a & \sigma_{1}
\end{array}\right]\left[\begin{array}{c}
\xi e_{i}^{T} \\
\eta e_{j}^{T}
\end{array}\right] \\
& =\frac{1}{\operatorname{det}\left(A_{11}\right)}\left(\sigma_{2} \xi^{2} e_{i} e_{i}^{T}-a \xi \eta e_{j} e_{i}^{T}+\sigma_{1} \eta^{2} e_{j} e_{j}^{T}-a \xi \eta e_{i} e_{j}^{T}\right) .
\end{aligned}
$$

Thus the only two off-diagonal elements of this matrix are in positions $(i, j)$ and $(j, i)$. Since $A$ is triadic, $A_{22}$ has at most one nonzero element in each of $i$ th and $j$ th rows, so the sum of $A_{22}$ and $A_{21} A_{11}^{-1} A_{21}^{T}$ is triadic.

THEOREM 2.4. In the $L B L^{T}$ factorization of a symmetric triadic matrix, $L$ is triadic.

Proof. Again the proof is by finite induction. At the $k$ th step, assume that the remaining matrix $\bar{A}$ is triadic. If the next pivot is $1 \times 1$, then Lemma 2.1 and the argument in the proof of Theorem 2.2 show that the next column of $L$ is triadic, as is the new remaining matrix. If the next pivot is $2 \times 2$, then the factorization produces

$$
\bar{A}=\left[\begin{array}{ll}
A_{11} & A_{21}^{T} \\
A_{21} & A_{22}
\end{array}\right]=\left[\begin{array}{cc}
I_{2} & 0 \\
A_{21} A_{11}^{-1} & I_{k-2}
\end{array}\right]\left[\begin{array}{cc}
A_{11} & 0 \\
0 & \tilde{A}
\end{array}\right]\left[\begin{array}{cc}
I_{2} & A_{11}^{-T} A_{21}^{T} \\
0 & I_{k-2}
\end{array}\right] .
$$

The off-diagonal part of the two new columns of $L$ is

$$
\begin{aligned}
A_{21} A_{11}^{-1} & =\frac{1}{\operatorname{det}\left(A_{11}\right)}\left[\begin{array}{ll}
\xi e_{i} & \eta e_{j}
\end{array}\right]\left[\begin{array}{cc}
\sigma_{2} & -a \\
-a & \sigma_{1}
\end{array}\right] \\
& =\frac{1}{\operatorname{det}\left(A_{11}\right)}\left[\begin{array}{cc}
\sigma_{2} \xi e_{i}-a \eta e_{j} & -a \xi e_{i}+\sigma_{1} \eta e_{j}
\end{array}\right],
\end{aligned}
$$

which is also triadic, and Lemma 2.3 shows that $\tilde{A}$ is triadic, so the induction can be continued.

Combining these results with the fact that the triadic property of a matrix is preserved under symmetric permutation, we see that the number of nonzero elements is $O(n)$ in all of these factorizations if diagonal pivoting is used. More precisely, by Lemmas 2.1 and 2.3, at most $n-2$ off-diagonal fill entries can occur.

THEOREM 2.5. If we factor a symmetric triadic matrix using any $L X L^{T}$ factorization with diagonal pivoting, then $L$ is triadic.

Although the columns of $L$ are sparse, the number of nonzero elements in each row of $L$ is bounded only by $n$; if $A$ is tridiagonal, for example, and

$$
\tilde{Z}=\left[\begin{array}{cccc}
0 & & & 1 \\
1 & \ddots & & \\
& \ddots & \ddots & \\
& & 1 & 0
\end{array}\right]
$$

is the circular shift-down matrix, then the last row of $L$ in the factorization $\tilde{Z}^{T} A \tilde{Z}=$ $L D L^{T}$ is generally full. 
3. Diagonal pivoting strategies for symmetric indefinite matrices. If the symmetric matrix $A \in R^{n \times n}$ is positive semidefinite [7], [15, section 10.3] or diagonally dominant [7], [13, section 9.5] (i.e., $\left|a_{i i}\right| \geq \sum_{j \neq i}\left|a_{i j}\right|$ for $i=1, \ldots, n$ ), then the largest magnitude element will appear on the diagonal. Each Schur complement inherits the property of positive semidefiniteness or diagonal dominance. Therefore, in either case, the elements of $L$ in the $L D L^{T}$ factorization are bounded in magnitude by 1 with pivoting. With or without pivoting, the growth factor for $D$ is $\rho(A)=1$ if $A$ is symmetric positive semidefinite, and $\rho(A) \leq 2$ if $A$ is diagonally dominant, where $\rho(A)$ is the ratio of the largest magnitude element in the Schur complements to the largest magnitude element in $A$.

We would like to compute factorizations of symmetric indefinite matrices that also give bounds on the elements of $L$ and $B$. In order to do this, it is necessary to pivot. There are three kinds of pivoting strategies in the literature: Bunch-Parlett [6] (complete pivoting); fast Bunch-Parlett and bounded Bunch-Kaufman [1] (rook pivoting); and Bunch-Kaufman [4] (partial pivoting). For full matrices, complete pivoting requires $O\left(n^{3}\right)$ comparisons, partial pivoting requires $O\left(n^{2}\right)$, and the cost of rook pivoting varies between $O\left(n^{2}\right)$ and $O\left(n^{3}\right)$. Therefore, it is important to uncover the advantages of the more expensive strategies. We consider each strategy in turn, applying each to the current Schur complement matrix $A$, noting that each depends on a preset constant $0<\alpha<1$.

3.1. Complete pivoting. Bunch and Parlett [6] devised the pivoting strategy presented in Algorithm 1.

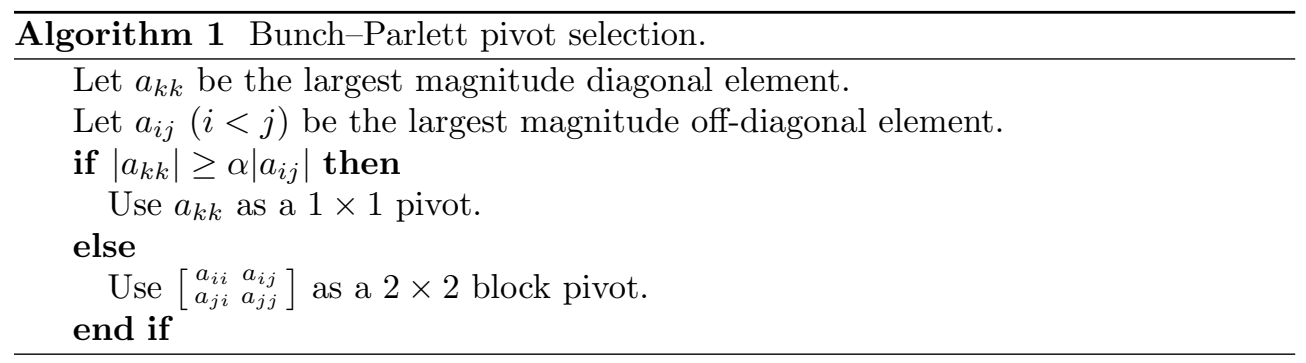

The process continues until $a_{k k}=a_{i j}=0$ or the factorization completes. The resulting pivot satisfies the following strong condition:

1. If a $1 \times 1$ pivot $a_{k k}$ is chosen, then $\left|a_{k k}\right| \geq \alpha\left|a_{p k}\right|$ for $p \neq k$.

2. If a $2 \times 2$ block pivot $\left[\begin{array}{cc}a_{i i} & a_{i j} \\ a_{j i} & a_{j j}\end{array}\right]$ is chosen, then each of the $1 \times 1$ pivots $a_{i i}$ and $a_{j j}$ satisfy $\left|a_{i i}\right|<\alpha\left|a_{i j}\right|$ and $\left|a_{j j}\right|<\alpha\left|a_{i j}\right|$, and $a_{i j}$ is the element of maximum magnitude in both column $i$ and column $j$.

For any algorithm satisfying the strong condition, the elements in $L$ are bounded and the element growth in $B$ during the factorization is well controlled, as we will show in section 3.5 .

3.2. Rook pivoting. The cost for finding a pivot satisfying the strong condition can be reduced by the iterative process in Algorithm 2 .

If the initial pivot index $i=1$, this is called a bounded Bunch-Kaufman pivot selection, while if $a_{i i}$ is the maximal magnitude diagonal element, it is called a fast Bunch-Parlett pivot selection [1]. Note that for a fast Bunch-Parlett selection, we do not need to test whether $a_{j j}$ is a $1 \times 1$ pivot, because if the initial maximum magnitude diagonal element $a_{i i}$ failed to be a pivot at the beginning, $\left|a_{j j}\right|$ is at most $\left|a_{i i}\right|$, and $\left|a_{i j}\right|$ is increasing in the loop. 


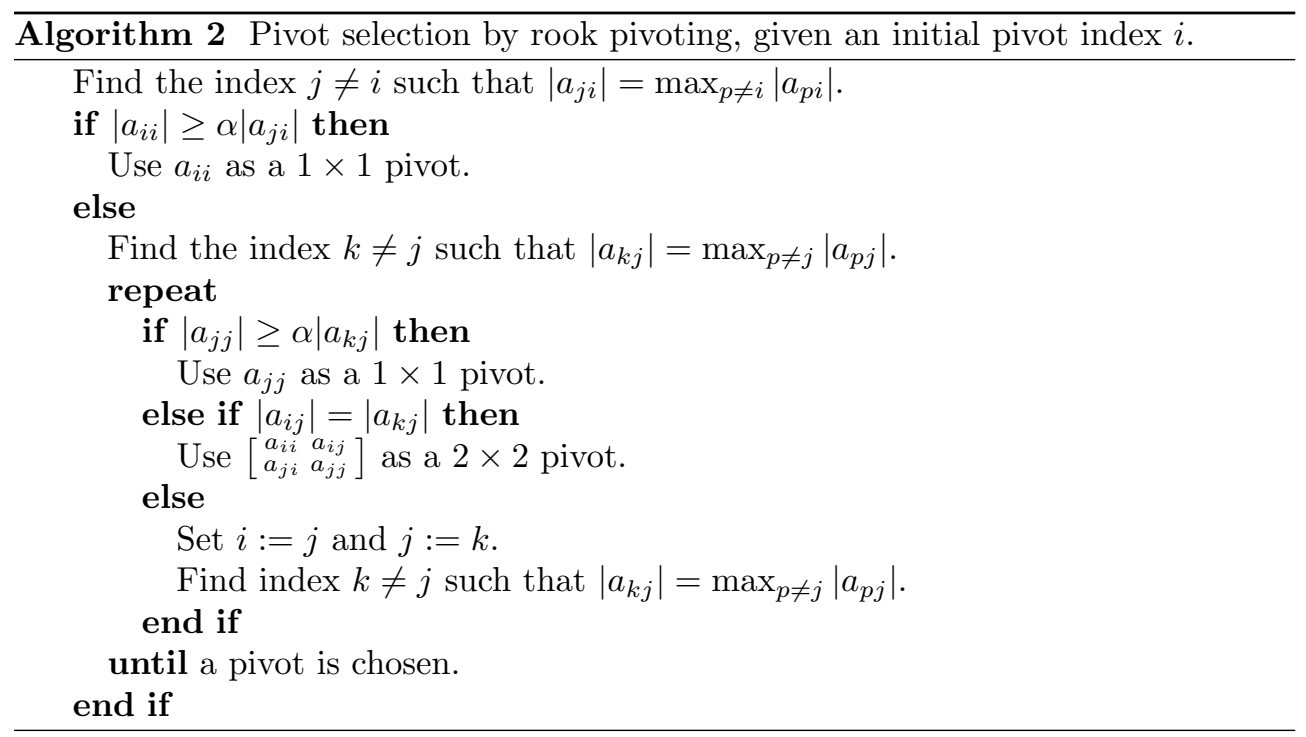

3.3. Partial pivoting. Bunch and Kaufman [4] devised the efficient pivoting strategy shown in Algorithm 3.

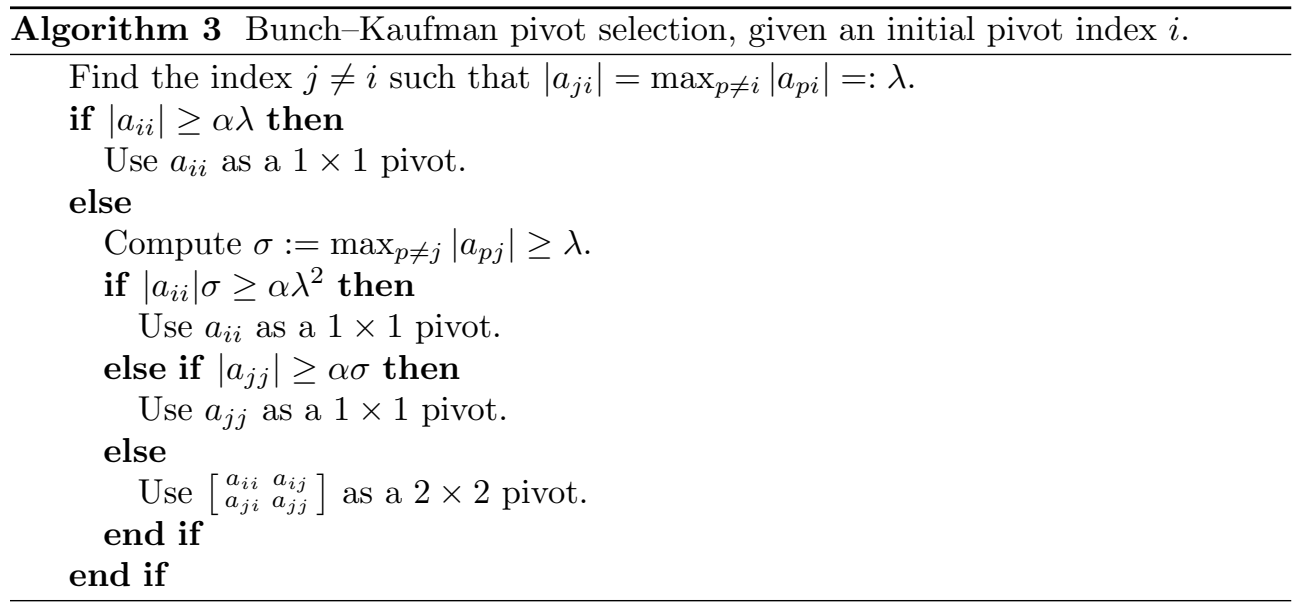

Bunch-Kaufman pivoting does not guarantee the strong condition, but satisfies the following weak condition:

1. If a $1 \times 1$ pivot $a_{k k}$ is chosen, then

- $\left|a_{k k}\right| \max _{p \neq q}\left\{\left|a_{p q}\right|:\left(a_{q k} \neq 0\right.\right.$ or $\left.\left.q=k\right)\right\} \geq \alpha \max _{p \neq k}\left|a_{p k}\right|^{2}$.

2. If a $2 \times 2$ block pivot $\left[\begin{array}{cc}a_{i i} & a_{i j} \\ a_{j i} & a_{j j}\end{array}\right]$ is chosen, then

- $\left|a_{i i}\right|<\alpha \lambda$,

- $\left|a_{i i}\right| \sigma<\alpha \lambda^{2}$

- $\left|a_{j j}\right|<\alpha \sigma$

where $\lambda=\max _{k \neq i}\left|a_{k i}\right|$ and $\sigma=\max _{k \neq j}\left|a_{k j}\right|$.

We compare the weak condition with the strong condition. For $1 \times 1$ pivots, $\max \left\{\left|a_{p q}\right|: p \neq q\right.$ and $\left(a_{q k} \neq 0\right.$ or $\left.\left.q=k\right)\right\} \geq \max _{p \neq k}\left|a_{p k}\right|$ so the strong condition guarantees the weak condition. For $2 \times 2$ block pivots, the weak condition meets the 
strong condition if $\sigma=\lambda$. We conclude that the strong condition implies the weak condition.

The natural choice of the initial pivot index $i$ in Algorithm 3 is $i=1$, which achieves the least cost to satisfy the weak condition [4].

Ashcraft, Grimes, and Lewis [1] argued that a bounded $L$ can improve stability. We can improve the probability that the Bunch-Kaufman algorithm has a bounded $L$ by choosing the largest magnitude diagonal entry as the search starting point at each pivot step [4]. The additional number of comparisons is $\frac{n^{2}}{2}+O(n)$, so the total comparison count remains $O\left(n^{2}\right)$. By making this change, we usually find a $1 \times 1$ pivot at the very first test at each step of pivot selection. The strong condition usually holds, but it is not guaranteed, as shown in the following example [13]:

$$
A=\left[\begin{array}{ccc}
\epsilon^{2} & \epsilon & \epsilon \\
\epsilon & 0 & 1 \\
\epsilon & 1 & 0
\end{array}\right]=\left[\begin{array}{ccc}
1 & & \\
\frac{1}{\epsilon} & 1 & \\
\frac{1}{\epsilon} & 0 & 1
\end{array}\right]\left[\begin{array}{ccc}
\epsilon^{2} & & \\
& -1 & \\
& & -1
\end{array}\right]\left[\begin{array}{ccc}
1 & \frac{1}{\epsilon} & \frac{1}{\epsilon} \\
& 1 & 0 \\
& & 1
\end{array}\right]=L B L^{T}
$$

where $L$ is unbounded as $\epsilon \rightarrow 0$.

3.4. The weak condition controls the growth factor. In summary, the Bunch-Parlett, fast Bunch-Parlett, and bounded Bunch-Kaufman pivoting strategies satisfy the strong condition, whereas the Bunch-Kaufman pivoting strategy and that of Ashcraft et al. satisfy the weak condition. The weak condition controls element growth during the factorization, as shown by an argument similar to those in $[1,4,6$, 13], [15, Chapter 11]. The growth factor in factoring $A \in R^{n \times n}$ is defined by

$$
\rho(A)=\frac{\max _{i, j, k}\left|a_{i j}^{(k)}\right|}{\max _{i, j}\left|a_{i j}\right|}
$$

where $a_{i j}$ and $a_{i j}^{(k)}$ are the $(i, j)$ entries of $A$ and of the $k$ th Schur complement, respectively, and $\|\cdot\|_{M}$ is the maximum magnitude element in the given matrix.

When a $1 \times 1$ pivot is chosen, we have

$$
\begin{aligned}
\frac{\max _{p \neq k}\left|a_{p k}\right|^{2}}{\left|a_{k k}\right|} & \leq \frac{1}{\alpha} \max \left\{\left|a_{p q}\right|: p \neq q \text { and }\left(a_{q k} \neq 0 \text { or } q=k\right)\right\} \\
& \leq \frac{1}{\alpha} \max _{p \neq q}\left|a_{p q}\right| .
\end{aligned}
$$

Therefore, the element growth is bounded by $1+\frac{1}{\alpha}$.

If a $2 \times 2$ block pivot is chosen, the weak condition guarantees $\left|a_{i i} a_{j j}\right|<\alpha^{2} \lambda^{2}$. Then

$$
\left|\operatorname{det}\left(\left[\begin{array}{cc}
a_{i i} & a_{i j} \\
a_{j i} & a_{j j}
\end{array}\right]\right)\right|=\left|a_{i j}^{2}-a_{i i} a_{j j}\right|>\left(1-\alpha^{2}\right) \lambda^{2} .
$$

Since $0<\alpha<1$,

$$
\left|\left[\begin{array}{cc}
a_{i i} & a_{i j} \\
a_{j i} & a_{j j}
\end{array}\right]^{-1}\right|<\frac{1}{\left(1-\alpha^{2}\right) \lambda^{2}}\left[\begin{array}{cc}
\left|a_{j j}\right| & \lambda \\
\lambda & \left|a_{i i}\right|
\end{array}\right]
$$


Therefore, the increase of each element in magnitude for the $2 \times 2$ block decomposition is bounded by

$$
\begin{aligned}
\frac{1}{\left(1-\alpha^{2}\right) \lambda^{2}}[\lambda \quad \sigma]\left[\begin{array}{cc}
\left|a_{j j}\right| & \lambda \\
\lambda & \left|a_{i i}\right|
\end{array}\right]\left[\begin{array}{l}
\lambda \\
\sigma
\end{array}\right] & =\frac{1}{\left(1-\alpha^{2}\right) \lambda^{2}}\left(\lambda^{2}\left(\left|a_{j j}\right|+\sigma\right)+\left(\lambda^{2}+\sigma\left|a_{i i}\right|\right) \sigma\right) \\
& <\frac{1}{\left(1-\alpha^{2}\right) \lambda^{2}}\left(\lambda^{2}(\alpha \sigma+\sigma)+\left(\lambda^{2}+\alpha \lambda^{2}\right) \sigma\right) \\
& =\frac{2(1+\alpha) \sigma}{1-\alpha^{2}}=\frac{2 \sigma}{1-\alpha},
\end{aligned}
$$

and the element growth for the $2 \times 2$ block decomposition is bounded by $1+\frac{2}{1-\alpha}$.

Therefore, element growth is bounded by

$$
g=\max \left\{1+\frac{1}{\alpha}, \sqrt{1+\frac{2}{1-\alpha}}\right\} .
$$

The minimum of $g$ is $\frac{1+\sqrt{17}}{2} \approx 2.562$, which is attained when $\alpha=\frac{1+\sqrt{17}}{8} \approx 0.640$.

Thus

$$
\rho(A) \leq g^{n-1} .
$$

The attainability of the last inequality is a research problem [15, Problem 11.10].

With complete pivoting (the Bunch-Parlett pivoting strategy), we can bound the growth factor of $A \in R^{n \times n}$ as

$$
\rho(A) \leq 3 n f(n), \quad \text { where } f(n)=\left(\prod_{k=2}^{n} k^{1 /(k-1)}\right)^{1 / 2} \leq 1.8 n^{(\ln n) / 4}
$$

with the pivoting argument $\alpha=\frac{1+\sqrt{17}}{8}$. This was shown by Bunch [2] with an analysis similar to Wilkinson's for Gaussian elimination with complete pivoting [16].

We note that the bounds on element increases in (3.2) and (3.4) are in terms of off-diagonal elements. Therefore, the growth factor $\bar{\rho}(A)$ for off-diagonal elements is bounded by $g^{n-2}$, i.e.,

$$
\bar{\rho}(A)=\frac{\max _{i \neq j, k}\left|a_{i j}^{(k)}\right|}{\max _{i \neq j}\left|a_{i j}\right|} \leq g^{n-2} \quad(n>1) .
$$

This is attainable, for example, with $\alpha=\frac{1+\sqrt{17}}{8}$ and

$$
A=\left[\begin{array}{ccccc}
-\alpha & 1 & 1 & \cdots & 1 \\
1 & -\alpha g-\frac{1}{\alpha} & 1 & \cdots & 1 \\
1 & 1 & -\alpha g^{2}-\frac{g}{\alpha}-\frac{1}{\alpha} & \ddots & \vdots \\
\vdots & \vdots & \ddots & \ddots & 1 \\
1 & 1 & \cdots & 1 & -\alpha g^{n-1}-\frac{g^{n-2}}{\alpha}-\frac{g^{n-3}}{\alpha}-\cdots-\frac{1}{\alpha}
\end{array}\right]
$$

The weak condition is stronger than necessary to bound the growth factor; we need only

$$
\left|a_{k k}\right| \max _{p \neq q}\left|a_{p q}\right| \geq \alpha \max _{p \neq k}\left|a_{p k}\right|^{2}
$$




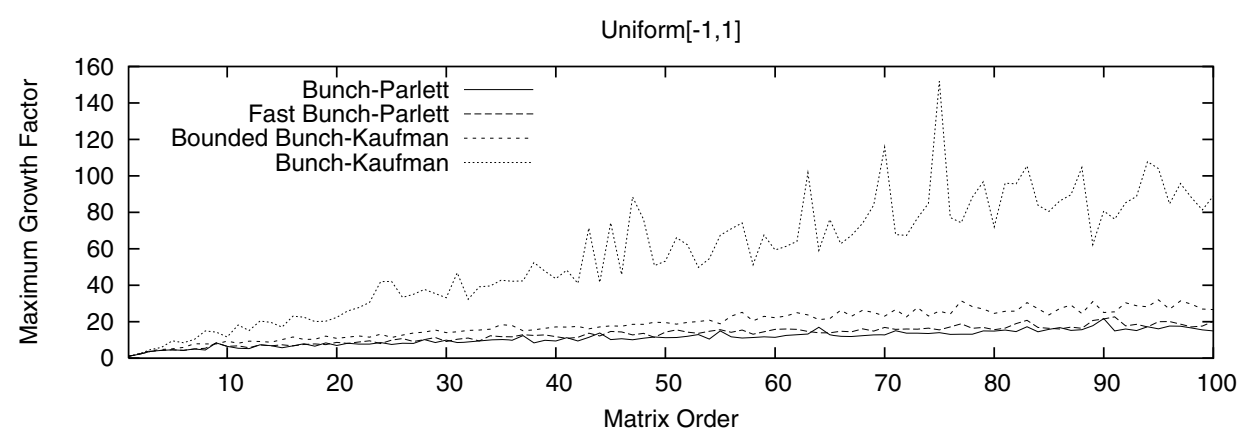

FIG. 3.1. Experimental maximum growth factor for factoring a symmetric matrix.

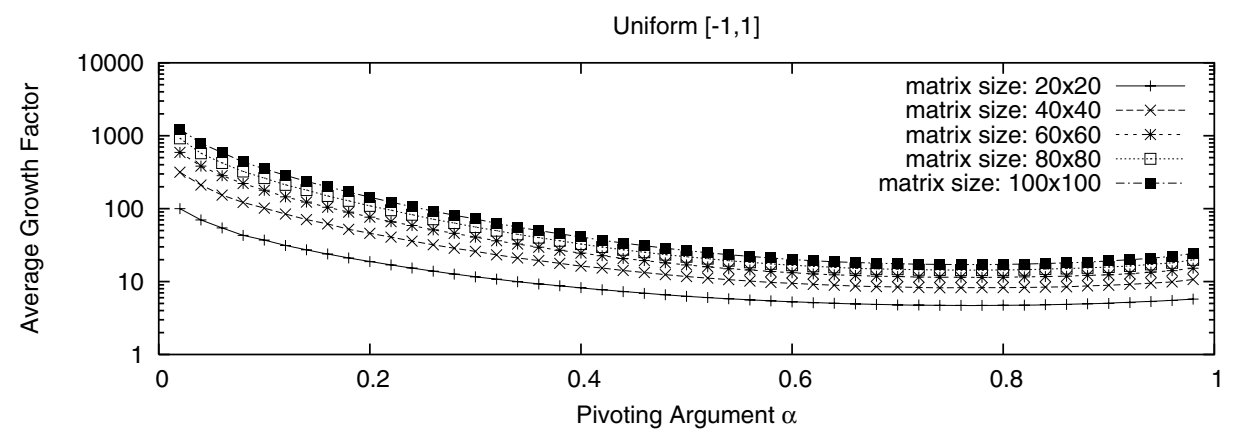

FIG. 3.2. Experimental average growth factor for factoring a symmetric matrix, Bunch-Kaufman.

for $1 \times 1$ pivots, but our version of the weak condition is useful for the triadic case considered in section 4.2 .

In practice, the average growth factors for both tridiagonal and full matrices are far from this bound. Figure 3.1 shows the maximum growth factor of 20,000 random symmetric $n \times n$ matrices for each $n=1, \ldots, 100$ with $\alpha=\frac{1+\sqrt{17}}{8} \approx 0.640$. In our experiments, all matrix elements are drawn independently from a uniform distribution on $[-1,1]$; results for a normal distribution are similar. Although $\alpha \approx 0.640$ minimizes the a priori bound on the growth factor, our experiments show that the best $\alpha$ to minimize the average growth factor with Bunch-Kaufman pivoting is usually between 0.74 and 0.78, as shown in Figure 3.2, where 20,000 random matrices are generated for each matrix size and each $\alpha$.

3.5. The strong condition bounds elements in $\boldsymbol{L}$. The weak condition does not bound $L$ for general matrices. For example [13], [15, section 11.1.2],

$$
A=\left[\begin{array}{lll}
0 & \epsilon & \\
\epsilon & 0 & 1 \\
& 1 & 1
\end{array}\right]=\left[\begin{array}{ccc}
1 & & \\
0 & 1 & \\
\frac{1}{\epsilon} & 0 & 1
\end{array}\right]\left[\begin{array}{ccc}
0 & \epsilon & \\
\epsilon & 0 & \\
& & 1
\end{array}\right]\left[\begin{array}{ccc}
1 & 0 & \frac{1}{\epsilon} \\
& 1 & 0 \\
& & 1
\end{array}\right]=L B L^{T}
$$

when the Bunch-Kaufman pivoting strategy is applied. As $\epsilon \rightarrow 0, L$ is unbounded.

In contrast, the strong condition does ensure a bound on elements in $L$. When a $1 \times 1$ pivot is chosen, then the magnitude of elements in the pivot column of $L$ is bounded by $\frac{1}{\alpha}$. If a $2 \times 2$ block pivot is chosen, the strong condition implies $\lambda=\sigma$ and 
TABLE 3.1

The element growth bound $g$ and the bound $\gamma$ for $L$ (when complete or rook pivoting is used) with two optimal choices of $\alpha$.

\begin{tabular}{||c|c|c|c||}
\hline & $\alpha$ & $\gamma$ & $g$ \\
\hline Minimize $g$ & $\frac{1+\sqrt{17}}{8} \approx 0.640$ & $\frac{7+\sqrt{17}}{4} \approx 2.781$ & $\frac{1+\sqrt{17}}{2} \approx 2.562$ \\
\hline Minimize $\gamma$ & $\frac{1}{2}$ & 2 & 3 \\
\hline
\end{tabular}

therefore the two columns of $L$ corresponding to this $2 \times 2$ block pivot have elements bounded by

$$
\begin{aligned}
\frac{1}{\left(1-\alpha^{2}\right) \lambda^{2}}\left[\begin{array}{ll}
\lambda & \sigma
\end{array}\right]\left[\begin{array}{cc}
\left|a_{j j}\right| & \lambda \\
\lambda & \left|a_{i i}\right|
\end{array}\right] & <\frac{1}{\left(1-\alpha^{2}\right) \lambda^{2}}\left[\begin{array}{ll}
\lambda & \lambda
\end{array}\right]\left[\begin{array}{cc}
\alpha \lambda & \lambda \\
\lambda & \alpha \lambda
\end{array}\right] \\
& =\frac{1+\alpha}{1-\alpha^{2}}\left[\begin{array}{ll}
1 & 1
\end{array}\right]=\frac{1}{1-\alpha}\left[\begin{array}{ll}
1 & 1
\end{array}\right] .
\end{aligned}
$$

Therefore, the elements in $L$ are bounded in magnitude by

$$
\gamma=\max \left\{\frac{1}{\alpha}, \frac{1}{1-\alpha}\right\}
$$

3.6. The growth factor and element bounds. We summarize the results on element growth in the following theorem, which extends some previous results to general $\alpha$.

TheOREM 3.1. For $L B L^{T}$ factorization of a symmetric matrix $A \in R^{n \times n}$, if the weak condition holds, then the growth factor $\rho(A)$ defined in (3.1) is bounded by

$$
\rho(A) \leq g^{n-1},
$$

where

$$
g=\max \left\{1+\frac{1}{\alpha}, \sqrt{1+\frac{2}{1-\alpha}}\right\},
$$

where $\alpha$ is the parameter in the factorization algorithm. If the strong condition holds, then the elements in $L$ are bounded in magnitude by

$$
\gamma=\max \left\{\frac{1}{\alpha}, \frac{1}{1-\alpha}\right\}
$$

As shown above, $\alpha=\frac{1+\sqrt{17}}{8}$ minimizes $g$, the element growth bound. But $\alpha=0.5$ minimizes the bound $\gamma$ on the elements of $L$. The consequences of each of these choices are summarized in Table 3.1.

4. Diagonal pivoting strategies for triadic symmetric matrices. In section 2, we showed that sparsity is preserved in the $L X L^{T}$ factorization of a symmetric triadic matrix with any diagonal pivoting strategy. In this section, we study a pivoting strategy particular to symmetric tridiagonal matrices [3] and also apply the pivoting strategies from the previous section to triadic matrices. 


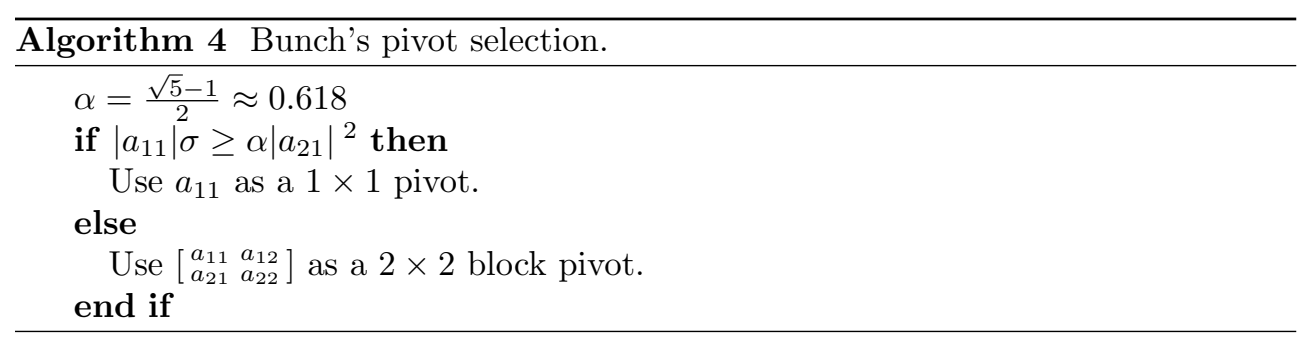

4.1. Pivoting strategies specific to symmetric tridiagonal matrices. One pivoting strategy has been proposed for $L B L^{T}$ factorizations of irreducible tridiagonal matrices. Consider the variant proposed by Higham [14] of the algorithm of Bunch [3] represented in Algorithm 4, with parameter $\sigma=\max _{i, j}\left|a_{i j}\right|$. The algorithm's great advantage is that there are no interchanges of rows and columns, yet the growth factor is bounded by

$$
\rho(A)=\max \left\{1+\frac{1}{\alpha}, \frac{1}{1-\alpha}\right\}
$$

whose minimum is achieved by choosing $\alpha=\frac{\sqrt{5}-1}{2}$. This method is excellent for applications relying on $B$ (e.g., computing inertia), but there is no element bound on $L$, illustrated, for example, as $\epsilon \rightarrow 0$ and

$$
A=\left[\begin{array}{cc}
\epsilon^{2} & \epsilon \\
\epsilon & 1
\end{array}\right]=\left[\begin{array}{ll}
1 & \\
\frac{1}{\epsilon} & 1
\end{array}\right]\left[\begin{array}{ll}
\epsilon^{2} & \\
& 0
\end{array}\right]\left[\begin{array}{cc}
1 & \frac{1}{\epsilon} \\
& 1
\end{array}\right] .
$$

A similar example is given in [14]. Therefore, this algorithm is not well suited to computing Newton-like directions or solving tridiagonal systems of equations with corner elements. Nevertheless, Higham showed that it is a stable method for solving linear symmetric tridiagonal systems [14].

Note that Algorithm 4 requires computing the maximum magnitude element $\sigma$ of the original matrix in advance. Recently Bunch and Marcia [5] developed another $L B L^{T}$ factorization algorithm for symmetric tridiagonal matrices that does not need the whole matrix a priori and requires no interchanges of rows and columns. It is favored in some applications.

4.2. Pivoting strategies from those for dense matrices. All the pivoting strategies from section 3 can be applied to a symmetric triadic matrix $A \in R^{n \times n}$. The growth factor is constrained because of the triadic structure, and we obtain a sharper result for $\rho(A)$ than that of Theorem 3.1, although the bound $\gamma$ on the elements of $L$ remains the same.

THEOREM 4.1. For $L B L^{T}$ factorization of a symmetric triadic $A \in R^{n \times n}$, consider the growth factor $\rho(A)$, defined in (3.1). If the weak condition holds,

$$
\rho(A) \leq \begin{cases}\frac{4 g\left(g^{(n-3) / 2}-1\right)}{g-1}+2\left(g^{(n-1) / 2}+g^{(n+1) / 2}\right)+1 & \text { if } n \text { odd }, \\ \frac{4 g\left(g^{(n-2) / 2}-1\right)}{g-1}+2 g^{n / 2}+1 & \text { if } n \text { even. }\end{cases}
$$

That is, $\rho(A)=O\left(g^{n / 2}\right)$. If the strong condition holds,

$$
\rho(A) \leq 2 n g^{\lfloor\lg (n-1)\rfloor} \leq 2 n(n-1)^{\lg g}=O\left(n^{1+\lg g}\right),
$$


where $n>1$ and $g=\max \left\{\frac{1}{\alpha}, \frac{1}{1-\alpha^{2}}\right\}$.

The proof of this theorem is given in the appendix.

If we choose $\alpha=\frac{\sqrt{5}-1}{2}$ to minimize $g$, then $\lg g \approx 0.694$, and therefore the bound for the strong condition is subquadratic. Even linear growth is rare, but it is possible; for example, if we take the circulant matrix $A$ with second row equal to $[1,-2,1,0, \ldots, 0]$ and change its $(1,1)$ element to -1 , then $\rho(A)=n / 2+O(1)$.

For the weak condition, exponential growth is achievable; define the $n \times n$ matrix

$$
A=\left[\begin{array}{ccccccccc}
-a & -1 & 0 & 1 & 0 & 0 & & \\
-1 & -a & 0 & 0 & 0 & 0 & & & 1 \\
0 & 0 & -a & -1 & 0 & 1 & & & 0 \\
1 & 0 & -1 & (g-1) a & 0 & 0 & & \\
0 & 0 & 0 & 0 & -a & -1 & & \ddots & \\
0 & 0 & 1 & 0 & -1 & (g-1) a & & & 0 \\
& & & & & & \ddots & \ddots & \vdots \\
& & & & \ddots & & \ddots & \ddots & 0 \\
0 & 1 & 0 & 0 & 0 & 0 & \cdots & 0 & 1
\end{array}\right],
$$

$|a|<\alpha=\frac{\sqrt{5}-1}{2}$ and $n$ odd. Then when $a \rightarrow \alpha^{-}$, the $(n, 2 j)$ entry becomes $g^{j-1}$ after $(j-1) 2 \times 2$ pivots for $j=1, \ldots, \frac{n-1}{2}$, and therefore $\rho(A)=O\left(g^{n / 2}\right)$.

Despite these examples, in our experiments, $\rho(A)$ is almost always bounded by a constant for both the weak and the strong conditions.

Although $\alpha=\frac{\sqrt{5}-1}{2} \approx 0.618$ minimizes the a priori bound on the relative element increase, our experiments show that the best $\alpha$ to minimize the average growth factor is usually between 0.82 and 0.86 for Bunch-Kaufman pivoting, as illustrated in Figure 4.1, where 20,000 random matrices are generated for each matrix size and $\alpha$.

With pivoting argument $\alpha=\frac{\sqrt{5}-1}{2}$, there are symmetric triadic matrices $A$ having $\rho(A)=O\left(g^{n / 2}\right)$ and $\rho(A)=O(n)$ for the weak and strong conditions, respectively. But our experiments show that, in practice, $L B L^{T}$ factorizations of symmetric tridiagonal or symmetric tridiagonal matrices with corner elements added usually show only constant growth in $\rho(A)$, whenever any of the four pivoting strategies are applied. Figure 4.2 shows the maximum growth factor of 20,000 random symmetric tridiagonal $n \times n$ matrices for each $n=50,100, \ldots, 1000$ and for random symmetric tridiagonal matrices with corner elements.

4.3. Pivoting cost. When the Bunch-Parlett algorithm is applied, it is natural to search the whole matrix instead of only the lower (or upper) triangular part due to the data structure for sparse matrices. So the number of comparisons is at most $3 k+O(1)$ to select a pivot in a $k \times k$ Schur complement. Therefore, the total number of comparisons is bounded by $\frac{3}{2} n^{2}+O(n)$ for a symmetric triadic $A \in R^{n \times n}$, which is more expensive than the $O(n)$ cost of the factorization. The Bunch-Kaufman algorithm requires at most $5 n+O(1)$ comparisons for a symmetric triadic $A \in R^{n \times n}$. For the fast Bunch-Parlett and bounded Bunch-Kaufman pivoting strategies, the worst-case number of comparisons is the same as that of Bunch-Parlett pivoting. The average number of element comparisons is between that for the Bunch-Kaufman and Bunch-Parlett pivoting strategies. Figure 4.3 shows the average number of comparisons of 20,000 symmetric matrices for each $n=50,100, \ldots, 1000$. 
Normal $\mathrm{N}(0,1)$

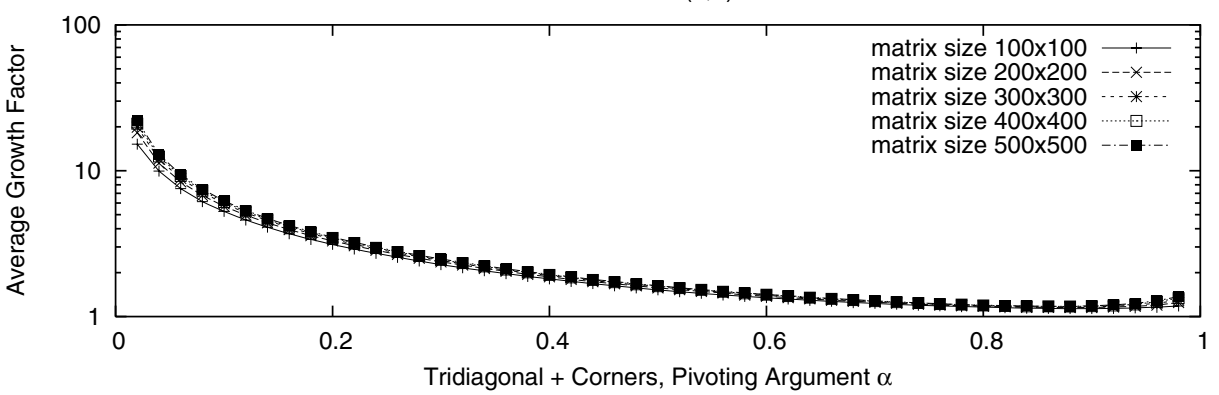

Uniform [-1,1]

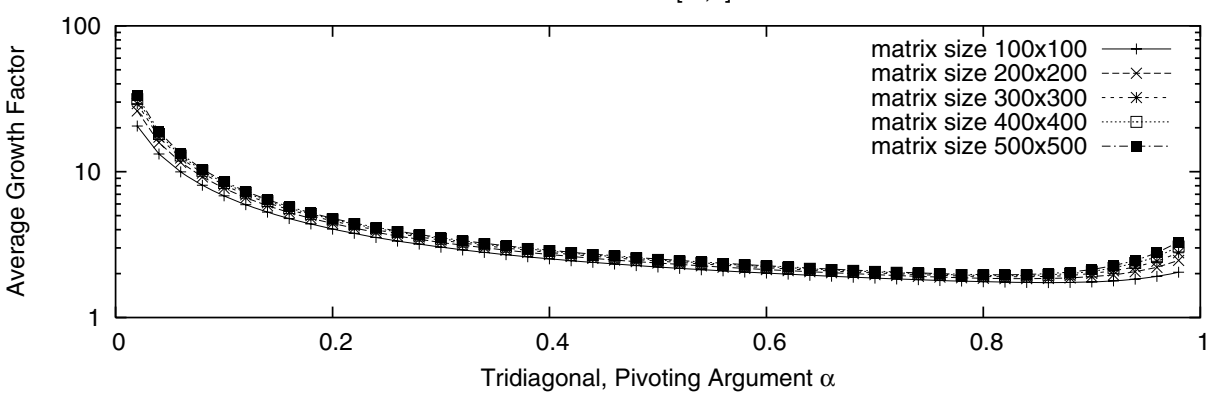

FIG. 4.1. Experimental average growth factor for Bunch-Kaufman pivoting on a symmetric triadic matrix or a symmetric tridiagonal matrix.

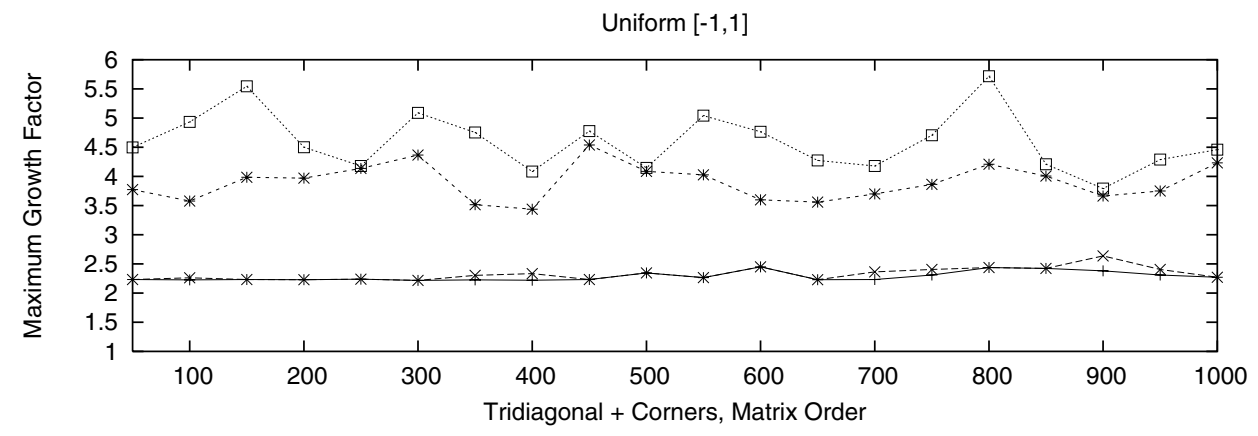

Uniform $[-1,1]$

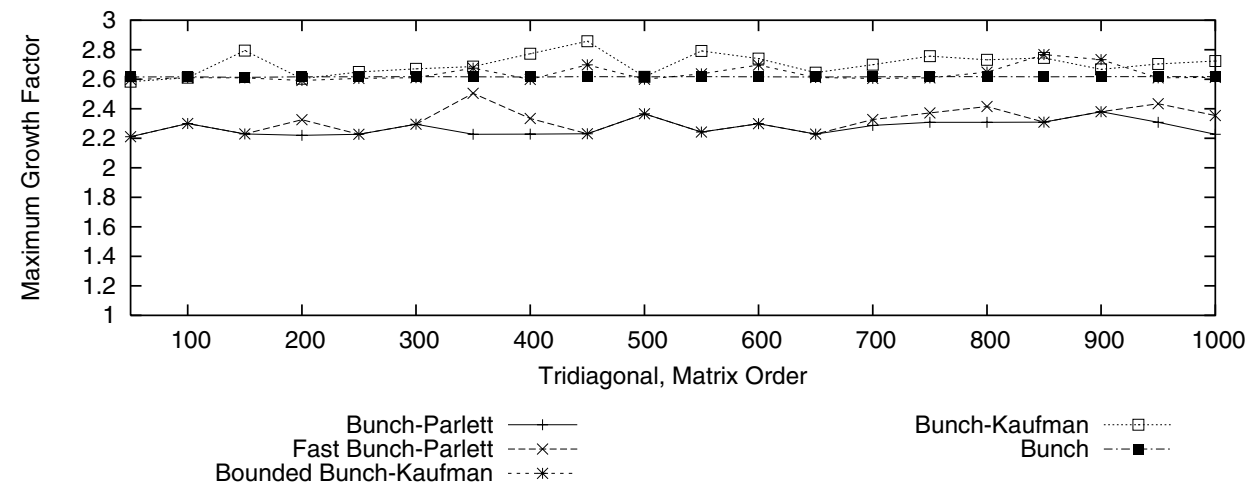

FIG. 4.2. Experimental average growth factor for factoring a symmetric triadic matrix or a symmetric tridiagonal matrix. 


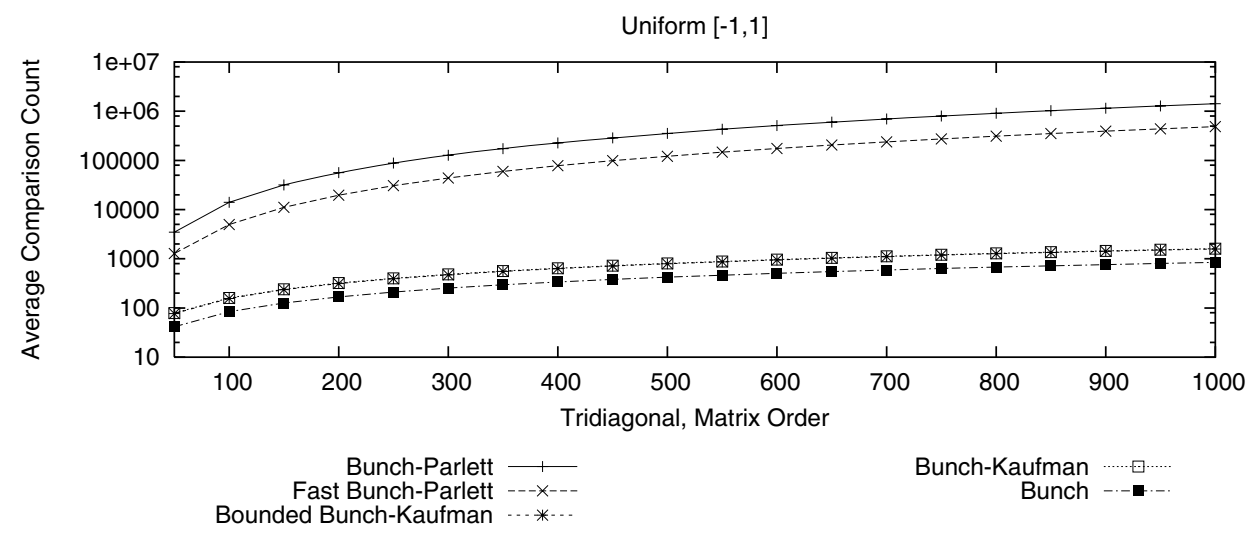

FIG. 4.3. Experimental average number of comparisons to factor a symmetric tridiagonal matrix.

5. Perturbation theory. The perturbation analysis of $L L^{T}$ factorization of a positive semidefinite symmetric matrix with complete pivoting is discussed in [12]. Partition $A$ as

$$
A=\left[\begin{array}{ll}
A_{11} & A_{21}^{T} \\
A_{21} & A_{22}
\end{array}\right]
$$

where $A_{11} \in R^{k \times k}$, and partition $L$ and $E$ accordingly. Assume that both $A_{11}$ and $A_{11}+E_{11}$ are nonsingular, and let $W=A_{11}^{-1} A_{21}^{T}=L_{11}^{-T} L_{21}^{T}$. In [12], Higham showed that with complete pivoting applied to a general positive semidefinite matrix,

$$
\|W\|_{2, F} \leq \sqrt{\frac{1}{3}(n-k)\left(4^{k}-1\right)} .
$$

We give bounds on $\|W\|_{2, F}$ for $L X L^{T}$ factorization of both full symmetric and symmetric triadic matrices.

TheOREM 5.1. Let $S_{k}(A)$ be the Schur complement appearing in an $L X L^{T}$ factorization of a symmetric matrix $A$ after processing the first $k$ columns and $k$ rows, $k<n$. Suppose there is a symmetric perturbation in A, denoted by E. Let $\|\cdot\|$ be a p-norm or the Frobenius norm and define absolute value $|\cdot|$ elementwise. Assume that both $A_{11}$ and $A_{11}+E_{11}$ are nonsingular. Then

$$
S_{k}(A+E)-S_{k}(A)=E_{22}-\left(E_{21} W+W^{T} E_{21}^{T}\right)+W^{T} E_{11} W+O\left(\|E\|^{2}\right),
$$

so

$$
\left|S_{k}(A+E)-S_{k}(A)\right| \leq\left|E_{22}\right|+\left|E_{21}\right||W|+\left|W^{T}\right|\left|E_{21}^{T}\right|+\left|W^{T}\right|\left|E_{11}\right||W|+O\left(\|E\|^{2}\right)
$$

and

$$
\left\|S_{k}(A+E)-S_{k}(A)\right\| \leq\|E\|\left(1+\|W\|^{2}\right)^{2}+O\left(\|E\|^{2}\right),
$$

where

$$
\|W\|_{2, F} \leq \sqrt{\frac{\gamma}{\gamma+2}(n-k)\left((1+\gamma)^{2 k}-1\right)}
$$


and $\gamma$ is a bound on the absolute value of the elements of $L$.

If $A$ is triadic, then this bound improves to

$$
\|W\|_{2, F} \leq \frac{2 \gamma \Phi_{\gamma}}{\Phi_{\gamma}-1} \sqrt{\frac{\Phi_{\gamma}^{2 k}-1}{\Phi_{\gamma}^{2}-1}}=O\left(\Phi_{\gamma}^{k}\right)
$$

where

$$
\Phi_{\gamma}=\frac{1+\sqrt{1+4 / \gamma}}{2} \gamma
$$

The proof of the theorem is contained in the following series of lemmas. We begin by generalizing to $L X L^{T}$ factorizations a result of Higham [12] for $L L^{T}$ factorization.

LEMma 5.2. Let $S_{k}(A)$ be the Schur complement appearing in an $L X L^{T}$ factorization of a symmetric matrix $A$ after processing the first $k$ columns and $k$ rows, $k<n$. Suppose there is a symmetric perturbation in $A$, denoted by E. Partition $A$ as

$$
A=\left[\begin{array}{ll}
A_{11} & A_{21}^{T} \\
A_{21} & A_{22}
\end{array}\right]
$$

where $A_{11} \in R^{k \times k}$, and partition $E$ accordingly. If $A_{11}$ and $A_{11}+E_{11}$ are nonsingular then

$$
S_{k}(A+E)=S_{k}(A)+E_{22}-\left(E_{21} W+W^{T} E_{21}^{T}\right)+W^{T} E_{11} W+O\left(\|E\|^{2}\right),
$$

where $W=A_{11}^{-1} A_{21}^{T}$.

Proof. The factorization takes the form

$$
A=\left[\begin{array}{ll}
A_{11} & A_{21}^{T} \\
A_{21} & A_{22}
\end{array}\right]=\left[\begin{array}{ll}
L_{11} & \\
L_{21} & I_{n-k}
\end{array}\right]\left[\begin{array}{ll}
X & \\
& S_{k}(A)
\end{array}\right]\left[\begin{array}{cc}
L_{11}^{T} & L_{21}^{T} \\
& I_{n-k}
\end{array}\right],
$$

where $L_{11} \in R^{k \times k}$ is lower triangular and the symmetric matrix $X \in R^{k \times k}$ is block diagonal with block order 1 or 2 . The matrix $X$ is either the identity, a diagonal matrix, or a block diagonal matrix, depending on the factorization. In any case, $A_{11}=L_{11} X L_{11}^{T}$ and $A_{21}=L_{21} X L_{11}^{T}$. Therefore, $W=A_{11}^{-1} A_{21}^{T}=L_{11}^{-T} L_{21}^{T}$. We also know that $S_{k}(A)=A_{22}-A_{21} A_{11}^{-1} A_{21}^{T}$, and since $A_{11}$ is nonsingular, $\left(A_{11}+E_{11}\right)^{-1}=$ $\left(I+A_{11}^{-1} E_{11}\right)^{-1} A_{11}^{-1}=\left(I-A_{11}^{-1} E_{11}\right) A_{11}^{-1}+O\left(\left\|E_{11}\right\|^{2}\right)$. The result is obtained by substituting the previous two equations into $S_{k}(A+E)=\left(A_{22}+E_{22}\right)-\left(A_{21}+\right.$ $\left.E_{21}\right)\left(A_{11}+E_{11}\right)^{-1}\left(A_{21}+E_{21}\right)^{T}$ and collecting the $O\left(\|E\|^{2}\right)$ terms.

Next, we bound the elements in $W=L_{11}^{-T} L_{21}^{T}$.

Lemma 5.3. If $L$ is unit lower triangular, with off-diagonal elements bounded in absolute value by $\gamma$, then

$$
|W|=\left|L_{11}^{-T} L_{21}^{T}\right| \leq y e^{T},
$$

where $y_{k-j}=\gamma(1+\gamma)^{j}$ and $e$ is a vector of ones.

Proof. The matrix $W$ satisfies $L_{11}^{T} W=L_{21}^{T}$, so let's consider a single column of this relationship. Let $r$ be a column of $W$. We will compute a vector $y$ satisfying $|r| \leq y$. Note that $\left|r_{k}\right| / \gamma$ is bounded by 1 , and $\left|r_{k-j}\right| / \gamma$ is bounded by 1 plus the sum of the later entries in $r$. If we let $s_{k-j}$ be a bound on the sum of the entries $k-j, \ldots, k$, then for $j=1,2, \ldots, k-1$, we have the recursions

$$
\begin{aligned}
& y_{k-j}=\gamma\left(1+s_{k-j+1}\right), \\
& s_{k-j}=s_{k-j+1}+y_{k-j},
\end{aligned}
$$


with $y_{k}=\gamma$ and $s_{k}=\gamma$. The solution to these recursions is

$$
\begin{aligned}
& y_{k-j}=\gamma(1+\gamma)^{j}, \\
& s_{k-j}=(1+\gamma)^{j+1}-1 .
\end{aligned}
$$

Therefore, each column of $W$ is bounded in absolute value by $y$ and the result follows.

The bound on $\|W\|$ follows immediately. This takes care of the general case and leaves only the triadic bound to be demonstrated. We begin with two simple lemmas and then proceed to the main result.

LEMmA 5.4. Let $F_{\gamma}(n)=\sum_{i=1}^{\lceil n / 2\rceil}\left(\begin{array}{c}n-i \\ i-1\end{array}\right) \gamma^{n-i}$ and $\Phi_{\gamma}=\frac{1+\sqrt{1+4 / \gamma}}{2} \gamma$. Then

$$
\frac{1}{1+(1 / \gamma)} \Phi_{\gamma}^{n-1} \leq F_{\gamma}(n) \leq \Phi_{\gamma}^{n-1}
$$

for $n=1,2, \ldots$ and $\gamma>0$.

Proof. We first observe that $F_{\gamma}(n)=\gamma\left(F_{\gamma}(n-1)+F_{\gamma}(n-2)\right)$ for $n>2$, with $F_{\gamma}(1)=1$ and $F_{\gamma}(2)=\gamma$. Note that $\gamma+\gamma \Phi_{\gamma}=\Phi_{\gamma}^{2}$. The result can be obtained by mathematical induction.

Lemma 5.5. Let $C \geq 0$ be an $m \times n$ matrix with $n \geq 2$. Then $\|C\|_{p} \leq\|C \hat{I}\|_{p}$, where $1 \leq p \leq \infty$ or $p=F$, and the $n \times(n-1)$ matrix $\hat{I}$ is the identity matrix of size $n-1$, with its last row repeated.

Proof. The cases of $p=F$ (Frobenius norm) and $p=\infty$ ( $\infty$-norm) are trivial. When $0 \leq p<\infty,\|C\|_{p}=\max _{\|x\|_{p}=1}\|C x\|_{p}=\|C z\|_{p}$, and this value is achieved for some $z$ with $\|z\|_{p}=1$. Note that $z_{i} \geq 0$ for $i=1, \ldots, n$, since all the elements of $C$ are nonnegative. Let $\hat{z}=\left[z_{1}, \ldots, z_{n-2}, \max \left(z_{n-1}, z_{n}\right)\right]^{T}$. Then $\|\hat{z}\|_{p} \leq 1$, and

$$
\|C\|_{p}=\|C z\|_{p} \leq\|C \hat{I} \hat{z}\|_{p} \leq\left\|C \hat{I}\left(\hat{z} /\|\hat{z}\|_{p}\right)\right\|_{p} \leq \max _{\|x\|_{p}=1}\|C \hat{I} x\|_{p}=\|C \hat{I}\|_{p} .
$$

Lemma 5.6. The $L B L^{T}$ factorization for symmetric triadic matrices has

$$
\left\|L_{11}^{-T} L_{21}^{T}\right\|_{2, F} \leq \frac{2 \gamma \Phi_{\gamma}}{\Phi_{\gamma}-1} \sqrt{\frac{\Phi_{\gamma}^{2 k}-1}{\Phi_{\gamma}^{2}-1}}=O\left(\Phi_{\gamma}^{k}\right),
$$

where $\gamma \geq 1$ is the off-diagonal element bound of $L$ and $\Phi_{\gamma}=\frac{1+\sqrt{1+4 / \gamma}}{2} \gamma$.

Proof. The proof of Lemma 5.4 shows $F_{\gamma}(i)=\gamma\left(F_{\gamma}(i-1)+F_{\gamma}^{2}(i-2)\right)$ for $i>2$. Observing the elements in $L_{11}^{-1} L_{11}=I$, we obtain

$$
\left|L_{11}^{-1}\right| \leq \sum_{i=1}^{k} F_{\gamma}(k) Z^{k-1}=\left[\begin{array}{ccccc}
F_{\gamma}(1) & & & & \\
F_{\gamma}(2) & F_{\gamma}(1) & & & \\
F_{\gamma}(3) & F_{\gamma}(2) & F_{\gamma}(1) & & \\
\vdots & \ddots & \ddots & \ddots & \\
F_{\gamma}(k) & \cdots & F_{\gamma}(3) & F_{\gamma}(2) & F_{\gamma}(1)
\end{array}\right]
$$

where $Z \in R^{k \times k}$ is the shift-down matrix. Note that this bound is attainable with $L_{11}=I-\gamma Z-\gamma Z^{2}$. By Lemma 5.4,

$$
\left|L_{11}^{-T}\right| e \leq\left[\frac{\Phi_{\gamma}^{k}-1}{\Phi_{\gamma}-1}, \frac{\Phi_{\gamma}^{k-1}-1}{\Phi_{\gamma}-1}, \ldots, 1\right]^{T} .
$$


Since $L$ is triadic, each row of $L_{21}^{T}$ has at most two nonzero elements. Let $\left|L_{21}^{T}\right|=$ $R_{1}+R_{2}$, where $R_{1}$ and $R_{2}$ contain the first and the second nonzero elements in each row, respectively. Then

$$
\left\|L_{11}^{-T} L_{21}^{T}\right\| \leq\left\|\left|L_{11}^{-T}\right|\left|L_{21}^{T}\right|\right\| \leq\left\|\left|L_{11}^{-T}\right| R_{1}\right\|+\left\|\left|L_{11}^{-T}\right| R_{2}\right\| .
$$

By Lemma 5.5,

$$
\begin{aligned}
\left\|\left|L_{11}^{-T}\right| R_{1}\right\| & \leq\left\|\left|L_{11}^{-T}\right| R_{1} \hat{I}_{n-k}\right\| \\
& \leq\left\|\left|L_{11}^{-T}\right| R_{1} \hat{I}_{n-k} \hat{I}_{n-k-1}\right\| \\
& \leq \cdots \leq\left\|\left|L_{11}^{-T}\right| R_{1} \hat{I}_{n-k} \hat{I}_{n-k-1} \cdots \hat{I}_{2}\right\| \\
& \leq\left\|\left|L_{11}^{-T}\right|(\gamma e)\right\|=\gamma\left\|L_{11}^{-T} \mid e\right\| .
\end{aligned}
$$

Similarly, $\left\|\left|L_{11}^{-T}\right| R_{2}\right\| \leq \gamma\left\|\left|L_{11}^{-T}\right| e\right\|$. By (5.1), for $\gamma \geq 1$

$$
\left\|L_{11}^{-T} L_{21}^{T}\right\|_{2, F} \leq 2 \gamma\left\|\left|L_{11}^{-T}\right| e\right\|_{2, F} \leq \frac{2 \gamma \Phi_{\gamma}}{\Phi_{\gamma}-1} \sqrt{\frac{\Phi_{\gamma}^{2 k}-1}{\Phi_{\gamma}^{2}-1}} .
$$

Note that this bound is halved when $n-k=1$.

For positive semidefinite triadic matrices and complete pivoting, $\gamma=1$ so $\Phi_{\gamma}^{k}=$ $O\left(\left(\frac{1+\sqrt{5}}{2}\right)^{k}\right)$.

In the $L B L^{T}$ factorization of a symmetric triadic matrix with diagonal pivoting, $\gamma$ can be 2 or $\frac{7+\sqrt{17}}{4} \approx 2.781$, to minimize the element bound of matrix $L$ or the element growth factor, respectively.

6. Concluding remarks. We have studied various pivoting strategies in computing the $L X L^{T}$ factorizations of symmetric triadic matrices. We denote the strategies as follows: BT (Bunch's pivoting strategy for a symmetric tridiagonal matrix), BP (Bunch-Parlett), FBP (fast Bunch-Parlett), BBK (bounded Bunch-Kaufman), and BK (Bunch-Kaufman). We summarize our results as follows:

1. The $L L^{T}, L D L^{T}$, and $L B L^{T}$ factors of a symmetric triadic matrix with any diagonal pivoting strategy remain sparse.

2. We have analyzed the boundedness of the growth factors in case the pivoting strategy satisfies either a strong or a weak condition.

3 . We have presented a new choice of the $\alpha$ parameter that better controls the growth factor.

4. In the $L B L^{T}$ factorization with various pivoting strategies, $L$ is bounded for $\mathrm{BP}, \mathrm{FBP}$, and BBK pivoting strategies, whereas the BK pivoting strategy may result in $L$ unbounded. All four pivoting strategies have the growth factor controlled for full symmetric matrices. The bound on the growth factor is smaller for symmetric triadic matrices.

5. For symmetric matrices, pivoting strategies BT and BK produce an $L$ matrix with no bounds on its elements, whereas the magnitude of elements in $L$ from pivoting strategies BBK, BP, and FBP is bounded by a constant $\gamma$ given in Table 3.1, depending on the parameter $\alpha$ in the algorithm.

6. For $L D L^{T}$ factorization of a positive definite symmetric matrix $A$ with complete pivoting, the magnification factor in the error bound for the Schur complement after $k$ steps is $\sqrt{1 / 3(n-k)\left(4^{k}-1\right)}$ if $A$ is full [12], and $O\left(\left(\frac{1+\sqrt{5}}{2}\right)^{k}\right)$ if $A$ triadic. 
7. For two pivoting strategies $D$ and $E$, we will say $D \succ E, D \succeq E$, and $D \simeq E$ if $D$ is better than, slightly better than, or similar to $E$, respectively. Our experimental results with pivoting argument $\alpha=\frac{\sqrt{5}-1}{2} \approx 0.618$ are as follows: For $L B L^{T}$ factorizations of uniformly distributed tridiagonal matrices, the maximum growth factors satisfy $\mathrm{BP} \succeq \mathrm{FBP} \succ \mathrm{BT} \succeq \mathrm{BBK} \succeq \mathrm{BK}$, as shown in Figure 4.2, whereas the average number of comparisons satisfies $\mathrm{BT} \succ$ $\mathrm{BK} \simeq \mathrm{BBK} \succ \mathrm{FBP} \succ \mathrm{BP}$, as shown in Figure 4.3. Thus, more expensive pivoting usually yields a smaller growth factor.

\section{Appendix. Proof of bounds for pivoting on triadics in Theorem 4.1.}

Theorem A.1. For $L B L^{T}$ factorization of a symmetric triadic $A \in R^{n \times n}$, the growth factor of off-diagonal elements $\bar{\rho}(A)$, defined in (3.6), is bounded as

$$
\bar{\rho}(A) \leq \begin{cases}2 g^{\lfloor\lg (n-1)\rfloor} \leq 2(n-1)^{\lg g} & \text { if strong condition holds } \\ 2 g^{\lfloor(n-1) / 2\rfloor} & \text { if weak condition holds }\end{cases}
$$

where $n>1$ and

$$
g=\max \left\{\frac{1}{\alpha}, \frac{1}{1-\alpha^{2}}\right\}
$$

Proof. Without loss of generality, we assume the required interchanges of rows and columns for pivoting are done prior to the factorization. Let $S_{k}(A)$ be the Schur complement of $A$ after reducing $k$ rows and $k$ columns, and let

$$
A^{(k+1)}=\underset{k}{k-k}\left[\begin{array}{cc}
k & n-k \\
0 & 0 \\
0 & S_{k}(A)
\end{array}\right] .
$$

By Lemmas 2.1 and 2.3, at most two diagonal and two off-diagonal elements are changed in the Schur complement. We denote them by $a_{i i}^{(k+1)}, a_{j j}^{(k+1)}, a_{i j}^{(k+1)}$, and $a_{j i}^{(k+1)}$. In addition to $a_{i j}^{(k+1)}$ and $a_{j i}^{(k+1)}, A^{(k+1)}$ has at most one nonzero off-diagonal element in each of $i$ th and $j$ th rows, inherited from $A^{(k-p)}$. Let $p=1$ or $p=2$ for the previous selection being $1 \times 1$ or $2 \times 2$, respectively.

Assume for now that

$$
a_{i j}^{(k+1-p)}=a_{j i}^{(k+1-p)}=0
$$

for each $k$. Later we will show that if this assumption breaks, the bounds on the off-diagonal growth factor are at most doubled.

For a $1 \times 1$ pivot, (A.1) implies that the weak condition coincides with the strong condition. Therefore,

$$
\left|a_{i j}^{(k+1)}\right|=\frac{\left|a_{i k}^{(k)}\right|\left|a_{j k}^{(k)}\right|}{\left|a_{k k}^{(k)}\right|} \leq \frac{1}{\alpha} \min \left\{\left|a_{i k}^{(k)}\right|,\left|a_{j k}^{(k)}\right|\right\} \leq g \min \left\{\left|a_{i k}^{(k)}\right|,\left|a_{j k}^{(k)}\right|\right\} .
$$

For a $2 \times 2$ pivot $\left[\begin{array}{cc}a_{k-1, k-1}^{(k-1)} & a_{k-1, k}^{(k-1)} \\ a_{k, k-1}^{(k-1)} & a_{k-1}^{(k-1)}\end{array}\right]$, there are at most two nonzero off-diagonal elements under the pivot, denoted by $a_{i, k-1}^{(k-1)}$ and $a_{j k}^{(k-1)}$. If $i=j$, then the only element changed in $A^{(k+1)}$ from $A^{(k-1)}$ is $a_{i i}^{(k+1)}$. In this case, the matrix size is 
reduced without increasing the off-diagonal elements. In order to maximize $\bar{\rho}(A)$, we assume $i \neq j$. The weak condition ensures (3.3). Therefore,

$$
\begin{aligned}
& \left|a_{i j}^{(k+1)}\right| \leq \frac{1}{\left(1-\alpha^{2}\right)\left|a_{k, k-1}^{(k-1)}\right|^{2}}\left[\left|a_{i, k-1}^{(k-1)}\right| \quad 0\right]\left[\begin{array}{cc}
\left|a_{k k}^{(k-1)}\right| & \left|a_{k-1, k}^{(k-1)}\right| \\
\left|a_{k, k-1}^{(k-1)}\right| & \left|a_{k-1, k-1}^{(k-1)}\right|
\end{array}\right]\left[\begin{array}{c}
0 \\
\left|a_{j k}^{(k-1)}\right|
\end{array}\right] \\
& =\frac{\left|a_{i, k-1}^{(k-1)}\right|\left|a_{j k}^{(k-1)}\right|}{\left(1-\alpha^{2}\right)\left|a_{k, k-1}^{(k-1)}\right|} \\
& \leq \begin{cases}g \min \left\{\left|a_{i, k-1}^{(k-1)}\right|,\left|a_{j k}^{(k-1)}\right|\right\} & \text { if strong condition holds, } \\
g\left|a_{j k}^{(k-1)}\right| & \text { if weak condition holds. }\end{cases}
\end{aligned}
$$

Since the Schur complement is symmetric, we consider the elements in the lower triangular. Let $G(m)=g^{m} \max _{i \neq j}\left|a_{i j}\right|$.

Consider the case that the strong condition holds. By (A.3) for a $2 \times 2$ pivot, an off-diagonal element of size $G(m)$ requires three $G(m-1)$ elements: $\left|a_{i, k-1}^{(k-1)}\right|,\left|a_{j k}^{(k-1)}\right|$, and $\left|a_{k, k-1}^{(k-1)}\right|$. Note that the strong condition guarantees $\left|a_{k, k-1}^{(k-1)}\right| \geq\left|a_{i, k-1}^{(k-1)}\right|$. By (A.2) for a $1 \times 1$ pivot, if $\left|a_{i j}^{(k+1)}\right| \geq G(m)$, then $\left|a_{i k}^{(k)}\right|,\left|a_{j k}^{(k)}\right| \geq G(m-1)$. In other words, for a $1 \times 1$ pivot, an off-diagonal element of size $G(m)$ requires two off-diagonal supporting elements of size $G(m-1)$. Therefore, the bound on element growth using $1 \times 1$ pivots is higher than that using $2 \times 2$ pivots. Note that each $1 \times 1$ elimination step introduces at most one fill-in entry. Considering a sequence of $2^{m-1}$ pivots of size $1 \times 1$, we see by induction that a $G(m)$ element requires $2^{m} G(0)$ elements that cannot contribute to the growth of other elements, and thus the growth must be logarithmic. We illustrate this in the following diagram for obtaining a $G(3)$ element with the smallest number of pivots. The last column indicates the Schur complements as the sources of the two off-diagonal elements in each row if they were not present initially. Note that $G(0)$ elements are from the original matrix $A$, whereas $G(1), G(2)$, and $G(3)$ elements are fill-in entries during the factorization.

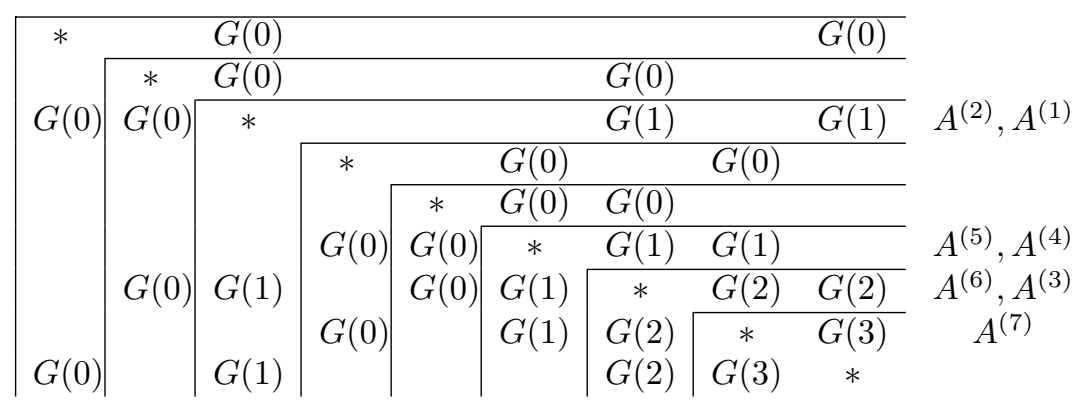

The number of pivots is $2^{m-1}+2^{m-2}+\cdots+2^{0}=2^{m}-1$. The last $2 \times 2$ Schur complement, with or without a row/column reduced afterward, cannot contribute to off-diagonal element growth. Therefore, the dimension of the smallest matrix that can have a $G(m)$ off-diagonal element is $\left(2^{m}-1\right)+2=2^{m}+1$. If $A$ has dimension less than $2^{m}+1$ but larger than $2^{m-1}$, then the off-diagonal elements in the Schur complements are at most $G(m-1)$ in magnitude. In other words,

$$
\bar{\rho}(A) \leq g^{\lfloor\lg (n-1)\rfloor} \leq(n-1)^{\lg g} .
$$

Consider the case that the weak condition holds. Recall that for a $1 \times 1$ pivot, the weak condition coincides with the strong condition, and an off-diagonal element of 
size $G(m)$ requires two $G(m-1)$ elements. By (A.3) for a $2 \times 2$ pivot, an off-diagonal element of size $G(m)$ requires only one $G(m-1)$ element. For maximal growth from $G(0)$ to $G(1)$ we use a $1 \times 1$ pivot. Otherwise, the bound on element growth using $2 \times 2$ pivots is at least as big as that using $1 \times 1$ pivots. The bound can increase by a factor of $g$ for every two rows reduced during the decomposition, except from $G(0)$ to $G(1)$ (one row/column reduced). The last Schur complement cannot contribute to off-diagonal element growth. Therefore,

$$
\bar{\rho}(A) \leq g^{\lfloor(n-1) / 2\rfloor},
$$

where $A \in R^{n \times n}$ is symmetric triadic.

So far we assume (A.1) holds. Now we show that if (A.1) breaks, the bounds in (A.4) and (A.5) are at most doubled. If $a_{i j}^{(k+1-p)}=a_{j i}^{(k+1-p)} \neq 0$, then there are no other off-diagonal elements in the $i$ th and $j$ th rows and columns in $A^{(k+1)}$, where $p=1,2$ stands for $1 \times 1,2 \times 2$ pivots, respectively. As a result, $A^{(k+1)}$ is a reducible matrix. After diagonally interchanging rows and columns, $A^{(k+1)}$ consists of two diagonal blocks: $\left[\begin{array}{cc}a_{i i}^{(k+1)} & a_{i j}^{(k+1)} \\ a_{j i}^{(k+1)} & a_{j j}^{(k+1)}\end{array}\right]$ and the remaining matrix, in which all the elements are taken from $A^{(k+1-p)}$. The bound on $a_{j i}^{(k+1)}$ in the $2 \times 2$ block is at most doubled, since it is a sum of two terms, each of which is bounded as (A.4) or (A.5), depending on whether the condition satisfied is strong or weak. Note that no off-diagonal element growth occurs afterward in this $2 \times 2$ block, and the other block is intact. Therefore, we obtain the result by safely declaring that the bounds in (A.4) and (A.5) are at most doubled if (A.1) breaks.

Theorem A.2. For $L B L^{T}$ factorization of a symmetric triadic $A \in R^{n \times n}$, consider the growth factor $\rho(A)$, defined in (3.1). If the weak condition holds,

$$
\rho(A) \leq \begin{cases}\frac{4 g\left(g^{(n-3) / 2}-1\right)}{g-1}+2\left(g^{(n-1) / 2}+g^{(n+1) / 2}\right)+1 & \text { if } n \text { odd }, \\ \frac{4 g\left(g^{(n-2) / 2}-1\right)}{g-1}+2 g^{n / 2}+1 & \text { if } n \text { even. }\end{cases}
$$

That is, $\rho(A)=O\left(g^{n / 2}\right)$. If the strong condition holds,

$$
\rho(A) \leq 2 n g^{\lfloor\lg (n-1)\rfloor} \leq 2 n(n-1)^{\lg g}=O\left(n^{1+\lg g}\right),
$$

where $n>1$ and $g=\max \left\{\frac{1}{\alpha}, \frac{1}{1-\alpha^{2}}\right\}$.

Proof. The major difference between $\rho(A)$ and $\bar{\rho}(A)$ is that the diagonal element increases can accumulate, whereas the accumulation of two off-diagonal element increases results in a reducible Schur complement, so further accumulation is impossible. Therefore, the diagonal element growth factor is bounded by the sum of $n$ elements, each of which is bounded by Theorem 4.1. So we obtain the bound on $\rho(A)$ for the strong condition. Though this approach also gives a bound for the weak condition, a tighter bound can be obtained, as follows.

The proof of Theorem A.1 shows that the off-diagonal element bound in the Schur complement depends on the number of rows/columns reduced. We follow the notation in the proof of Theorem A.1.

If the weak condition holds, the off-diagonal elements $a_{i j}^{(k+1)}$ in $A^{(k+1)}$ (after reducing $k$ rows/columns) are bounded as $\left|a_{i j}^{(k+1)}\right| \leq 2 g^{\lfloor(k+1) / 2\rfloor} \max \left|a_{i j}\right|$ for $i \neq j$ and $k$ from 1 to $n-2$. This is also the bound on the diagonal element increase of $A_{k+1}$ from the previous iteration. We sum up all the relative element increases during 
the decomposition to obtain a bound on $\rho(A)$, where $A \in R^{n \times n}$ is symmetric triadic:

$$
\begin{aligned}
\rho(A) & \leq \underline{1}+2 g^{\lfloor 2 / 2\rfloor}+2 g^{\lfloor 3 / 2\rfloor}+\cdots+2 g^{\lfloor(n-1) / 2\rfloor}+2 \underline{g^{\lfloor(n-1) / 2\rfloor+1}} \\
& = \begin{cases}\frac{4 g^{\left(g^{(n-3) / 2}-1\right)}+2\left(g^{(n-1) / 2}+g^{(n+1) / 2}\right)+1}{\text { if } n \text { odd },} \\
\frac{4 g\left(g^{(n-2) / 2}-1\right)}{g-1}+2 g^{n / 2}+1 & \text { if } n \text { even. }\end{cases}
\end{aligned}
$$

The underlined 1 occurs because each diagonal element in the initial $A$ can be $G(0)$. The reason for the last term $2 g^{\lfloor(n-1) / 2\rfloor+1}$ is as follows. If a $1 \times 1$ pivot is chosen in the last $2 \times 2$ Schur complement or a $2 \times 2$ pivot is chosen in the last $3 \times 3$ Schur complement, the reduction can still increase the very last diagonal element, but there is no off-diagonal element growth. If (A.1) breaks, the reduced $2 \times 2$ block can have diagonal element growth but no off-diagonal element growth. This case is also taken into account in $2 g^{\lfloor(n-1) / 2\rfloor+1}$. In a similar vein, we can also obtain a slightly tighter bound for the strong condition, but it is also $O\left(n^{1+\lg g}\right)$ :

$$
\rho(A) \leq 1+2 g^{\lfloor\lg 2\rfloor}+2 g^{\lfloor\lg 3\rfloor}+\cdots+2 g^{\lfloor\lg (n-1)\rfloor}+2 g^{\lfloor\lg (n-1)\rfloor+1}=O\left(n^{1+\lg g}\right) .
$$

Acknowledgments. The authors are grateful to the referees for their careful reading of the manuscript. It is a pleasure to thank Nick Higham for his encouragement in this work.

\section{REFERENCES}

[1] C. Ashcraft, R. G. Grimes, and J. G. Lewis, Accurate symmetric indefinite linear equation solvers, SIAM J. Matrix Anal. Appl., 20 (1998), pp. 513-561.

[2] J. R. Bunch, Analysis of the diagonal pivoting method, SIAM J. Numer. Anal., 8 (1971), pp. $656-680$.

[3] J. R. Bunch, Partial pivoting strategies for symmetric matrices, SIAM J. Numer. Anal., 11 (1974), pp. 521-528.

[4] J. R. Bunch And L. Kaufman, Some stable methods for calculating inertia and solving symmetric linear systems, Math. Comp., 31 (1977), pp. 163-179.

[5] J. R. Bunch And R. F. Marcia, A pivoting strategy for symmetric tridiagonal matrices, Numer. Linear Algebra Appl., 12 (2005), pp. 911-922.

[6] J. R. Bunch And B. N. Parlett, Direct methods for solving symmetric indefinite systems of linear equations, SIAM J. Numer. Anal., 8 (1971), pp. 639-655.

[7] J. W. Demmel, N. J. Higham, and R. S. Schreiber, Block LU factorization, Numer. Linear Algebra Appl., 2 (1995), pp. 173-190.

[8] H.-R. FANG, Backward Error Analysis of Factorization Algorithms for Symmetric and Symmetric Triadic Matrices, Tech. Report CS-4734, Computer Science Department, University of Maryland, College Park, MD, 2005.

[9] H.-R. Fang And D. P. O'Leary, Modified Cholesky Factorizations: A Catalog with New Approaches, Tech Report TR-4807, Computer Science Department, University of Maryland, College Park, MD, 2006.

[10] A. Forsgren, P. E. Gill, and W. Murray, Computing modified Newton directions using a partial Cholesky factorization, SIAM J. Sci. Comput., 16 (1995), pp. 139-150.

[11] P. E. Gill, W. Murray, And M. H. Wright, Unconstrained methods, in Practical Optimization, Academic Press, 1981, Chap. 4, pp. 105-116.

[12] N. J. Higham, Analysis of the Cholesky decomposition of a semi-definite matrix, in Reliable Numerical Computation, M. G. Cox and S. J. Hammarling, eds., Oxford University Press, New York, 1990, pp. 161-185.

[13] N. J. Higham, Stability of the diagonal pivoting method with partial pivoting, SIAM J. Matrix Anal. Appl., 18 (1997), pp. 52-65.

[14] N. J. Higham, Stability of block $L D L^{T}$ factorization of a symmetric tridiagonal matrix, Linear Algebra Appl., 287 (1999), pp. 181-189.

[15] N. J. Higham, Accuracy and Stability of Numerical Algorithms, 2nd ed., SIAM, Philadelphia, 2002.

[16] J. H. Wilkinson, Error analysis of direct methods of matrix inversion, J. ACM, 8 (1961), pp. $281-330$. 